\title{
Editorial
}

\section{Advanced Dynamic Simulations in Transportation}

\author{
Wei Guan, ${ }^{1}$ Xuedong Yan, ${ }^{1}$ Essam Radwan, ${ }^{2}$ Sze Chun Wong, ${ }^{3}$ and Xiaoliang Ma ${ }^{4}$ \\ ${ }^{1}$ MOE Key Laboratory for Urban Transportation Complex Systems Theory and Technology, Beijing Jiaotong University, \\ Beijing 100044, China \\ ${ }^{2}$ Center for Advanced Transportation Systems Simulations, University of Central Florida, Orlando, FL 32816, USA \\ ${ }^{3}$ Department of Civil Engineering, The University of Hong Kong, Pokfulam, Hong Kong \\ ${ }^{4}$ Department of Transportation Sciences and Centre for Traffic Research (CTR), Royal Institute of Technology (KTH), \\ 10044 Stockholm, Sweden
}

Correspondence should be addressed to Wei Guan; weig@bjtu.edu.cn

Received 6 July 2015; Accepted 7 July 2015

Copyright (C) 2015 Wei Guan et al. This is an open access article distributed under the Creative Commons Attribution License, which permits unrestricted use, distribution, and reproduction in any medium, provided the original work is properly cited.

Advanced simulation methods have been widely applied in the field of transportation for driving behavior investigation, planning, design and operation of transportation systems, evaluation of transportation policy, and innovation of intelligent transportation systems (ITS). With the development of computer technologies, dynamic simulations even in a real time manner become more and more powerful and sophisticated in either practical applications or scientific research in the transportation field. This special issue aims to discuss and share experience in theoretical analyses, state-ofthe-art techniques, and cutting-edge case studies of advanced dynamic transportation simulations.

In this special issue, we collect seven papers that involve different aspects of advanced simulation techniques to explore the mechanism of transportation operation, effectiveness of intelligent transportation systems, driving behavior and traffic operation, and transportation logistics system performance under various scenarios.

In the field of transportation logistics, closed-loop supply chain distribution network system has become a new research point since people pursue circular economy and social sustainable development. X. Yuan and X. Zhang establish use of Vensim software to simulate three-loop supply chain distribution network system model and investigate the relationship among supplier, manufacturer, retailers, and products (parts) recycler with the government policy. In a competitive environment, transportation costs are an important part of the costs of logistics enterprises.
Q. Yan and Q. Zhang present a biobjective transportation cost model for solving a multiobjective vehicle routing problem with soft time-window constraints that specify the earliest and latest arrival times of customers.

Traffic flow big data strongly shows temporal, spatial, and historical correlations. H. Lu et al. use speed and occupancy data to develop the traffic flow state clustering model. Using a bilevel optimization model, the authors calculate the number of temporal-spatial-historical correlation variables and traffic flow state formulation of regional traffic flow. Traffic sensors (e.g., magnetic detectors, cameras, and bluetooth detectors) provide real-time data for traffic operation surveillance and arterial travel time estimation. Considering the restriction in the sensors' installation cost, Q. Yu et al. propose a sensor location model to find the optimal sensor placement for minimizing the travel time estimation error in signalized arterial.

As the economy develops rapidly, deepening urbanization and the increasing urban population lead to a large demand for urban rail transit in many cities. The modern streetcar system is considered as an important mode of urban transit systems in China. However, its construction and development face massive challenges due to the conflicts between streetcars and other vehicles at the intersections in urban road networks. B. Wang et al. develop a segmented signal progression model for modern streetcar system in order to ensure high streetcar system efficiency and minimize its negative impacts on transit and vehicular traffic. 
On the other hand, many urban rail systems have been plagued by heavy passenger flow, and thus it is critical to design an efficient and economical timetable for a heavily congested urban rail corridor. Y.-T. Zhu et al. propose a model for timetable design of urban rail line with capacity constraints to identify the departure time of trains at the start terminal and minimize the system cost, including passenger waiting cost and operating cost. A two-stage simulationbased genetic algorithm is developed to solve the model.

Interestingly, while most of the simulation models are explored for motor vehicles, Zhou et al. improve the NagelSchreckenberg (NS) CA model and the multivalue CA (MCA) model for both regular bicycles and electric bicycles and provide useful simulation tools for mixed traffic operation analyses. A two-stage simulation-based genetic algorithm is developed to solve the model. In this study, different bicycle driving behaviors with characteristics of the slowing down probability and lane-changing probability were simulated using the proposed models. The results show that the M-CA model exhibits more stable performance than the two-lane NS model and provides results that are closer to real bicycle traffic performance.

Transportation simulation is a multidisciplinary area. The editors acknowledge that this special issue cannot fully cover all cutting-edge technologies and methods emerging in this field. However, these papers reveal both the promise and the challenges faced by the field of dynamic simulations in transportation. Through this special issue, we encourage more transportation researchers, educators, students, practitioners, and policy makers to seek and explore advanced dynamic simulation techniques to enhance transportation operation, safety, and environment quality.

\section{Acknowledgments}

We sincerely acknowledge all of the authors and anonymous reviewers' great contributions to this special issue.

Wei Guan

Xuedong Yan

Essam Radwan

Sze Chun Wong

Xiaoliang Ma 


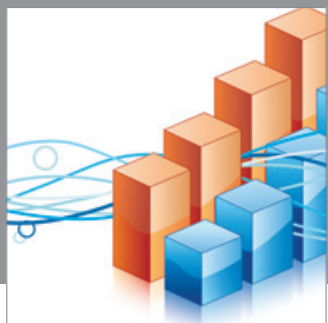

Advances in

Operations Research

mansans

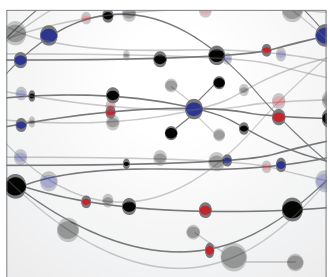

The Scientific World Journal
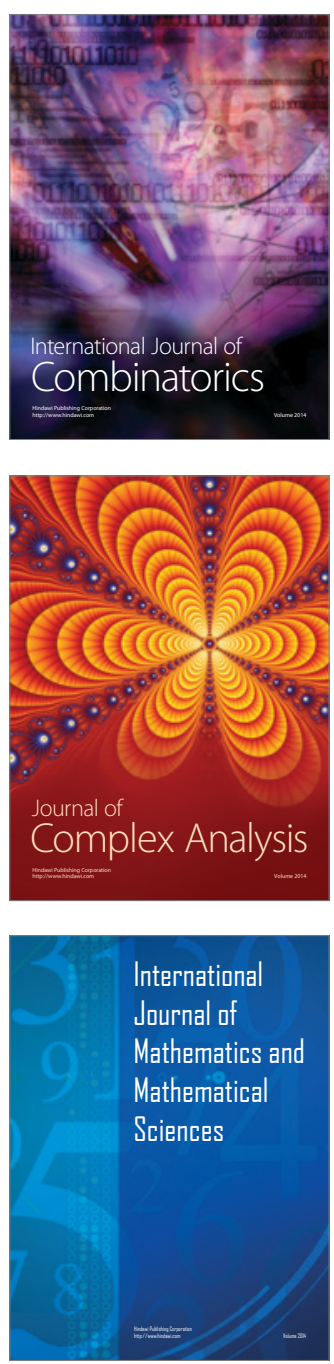
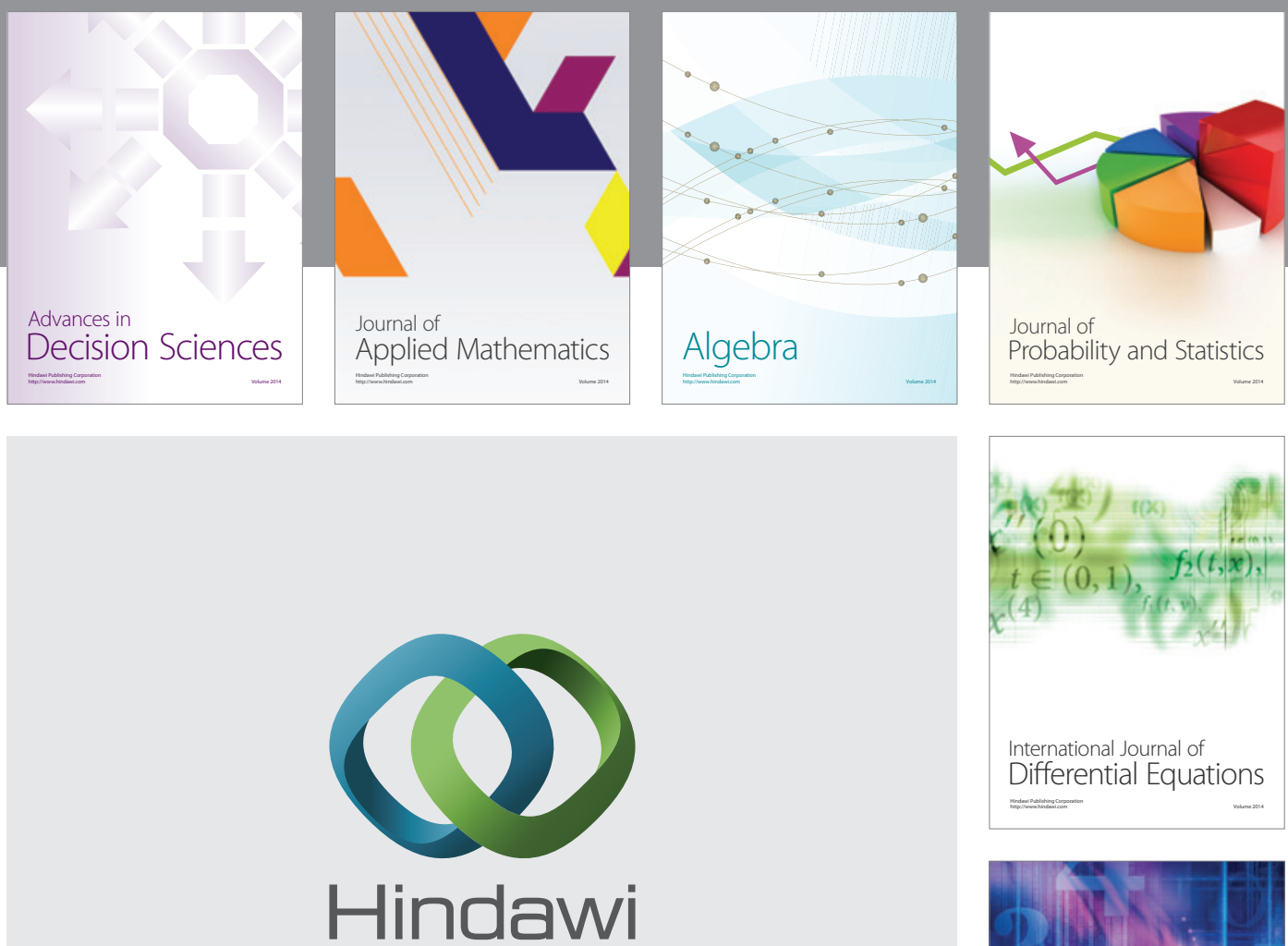

Submit your manuscripts at http://www.hindawi.com
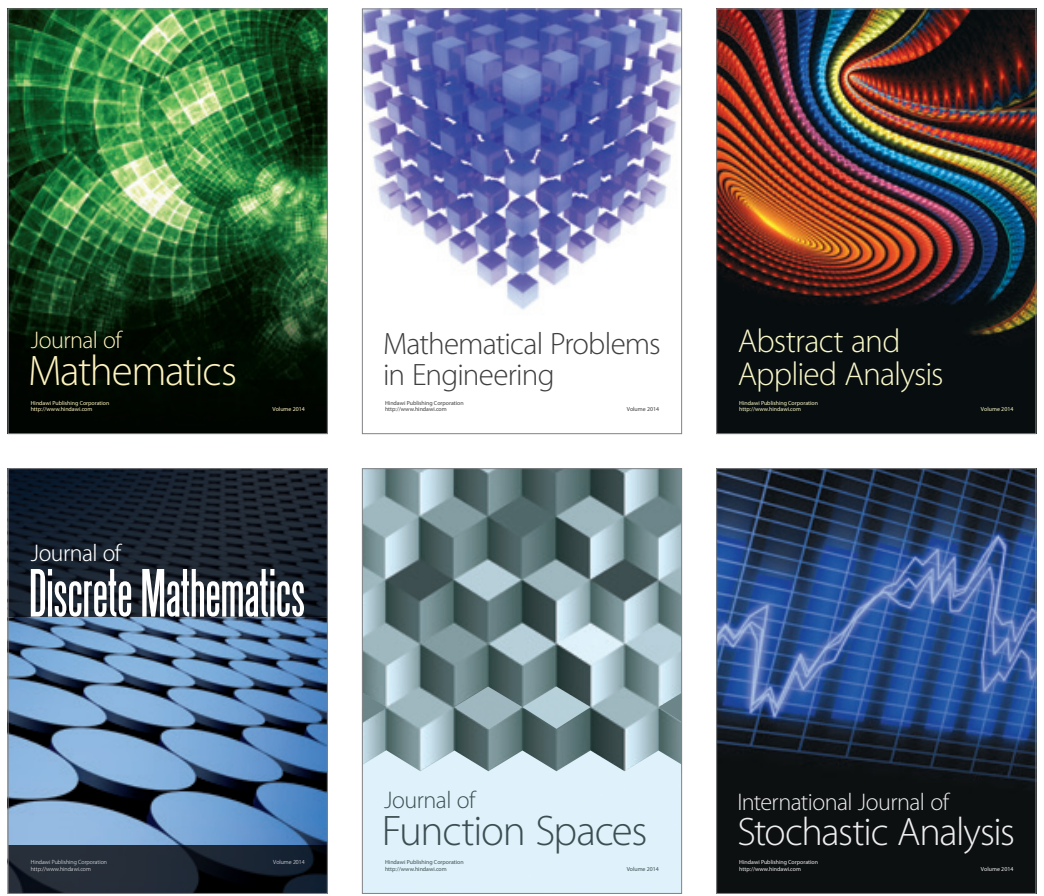

Journal of

Function Spaces

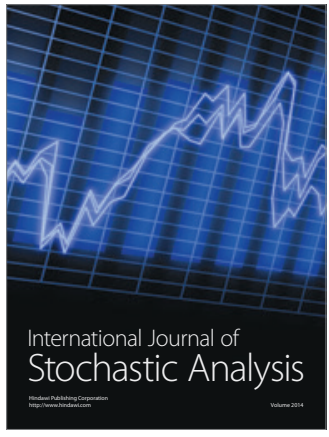

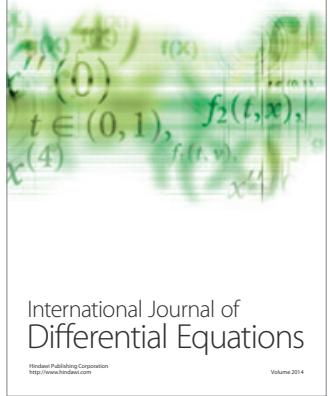
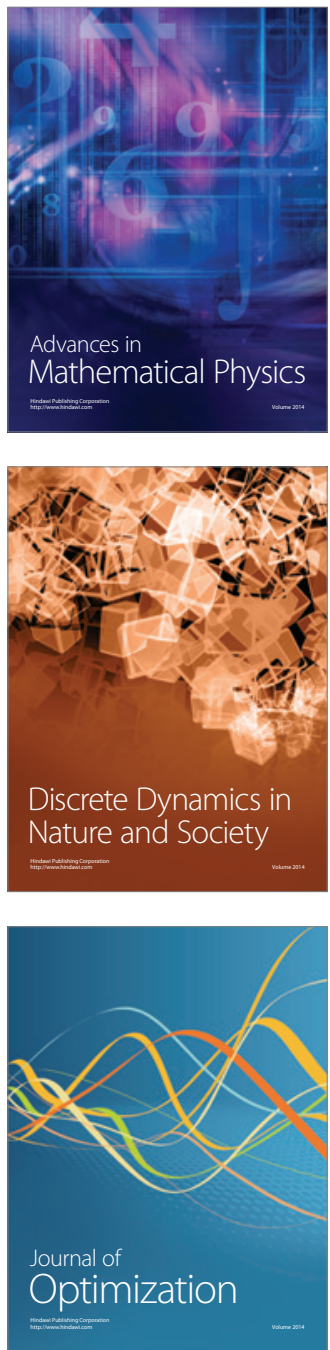